\title{
Fibrogenesis imperfecta ossium: an acquired incapacitating bone disease
}

\author{
Agueda Prior-Español ${ }^{1}$ (D) - Susana Holgado-Pérez ${ }^{1}$ (D) Alejandro Olivé $^{1}$ (D) $\cdot$ Laia Gifre $^{1}$ (D)
}

Received: 5 February 2021 / Revised: 15 March 2021 / Accepted: 18 March 2021 / Published online: 24 March 2021

(C) International League of Associations for Rheumatology (ILAR) 2021

\section{Presentation}

A 73-year-old man was referred to our Rheumatology Department with a 13 years history of incapacitating bone pain, mostly involving lower extremities and lumbar spine. He had bilateral tibia and fibula fractures and marked bone deformities. He had not received previous treatment with calcium, vitamin $\mathrm{D}$, or bisphosphonates. And he had no family history of bone disorder. Height was $168 \mathrm{~cm}$. Biochemical parameters of bone metabolism were normal: calcium $8.8 \mathrm{mg} / \mathrm{dL}$, phosphate $3.0 \mathrm{mg} / \mathrm{dL}$, alkaline phosphatase $105 \mathrm{U} / \mathrm{L}$, TSH $1.54 \mu \mathrm{UI} / \mathrm{mL}$, PTH $34.8 \mathrm{pg} / \mathrm{mL}$, calcidiol $31.5 \mathrm{ng} / \mathrm{mL}$, calciuria $78 \mathrm{mg} / 24 \mathrm{~h}$, phosphaturia $494 \mathrm{mg} / 24 \mathrm{~h}$. Paraproteinemia and BenceJones proteinuria were found. Bone marrow biopsy, thoracoabdominal tomography, serial bone radiographs, and subcutaneous fat biopsy were performed to rule out multiple myeloma, leukemia, lymphoma, and amyloidosis. Radiological studies showed fractures at multiple vertebrae, ribs, bilateral distal femur, and proximal tibia with fracture-related deformities (Fig. 1a-e). Bone scintigraphy revealed multiple uptake locations with diffuse skeletal involvement (Fig. 1f). Bone densitometry showed femoral neck osteopenia. Undecalcified trans-iliac bone biopsy, previously marked with tetracyclines, showed features of osteomalacia (Fig. 1g). Polarized light microscopy revealed disorganized lamellar bone structure and irregular collagen pattern (Fig. 1h), a finding that confirmed the diagnosis of fibrogenesis imperfecta ossium (FIO) [1]. Treatment with melphalan, glucocorticoids, and bisphosphonates had no effect on clinical, laboratory,

Agueda Prior-Español

agueda88@gmail.com

1 Rheumatology Department, Hospital Universitari Germans Trias i Pujol, Carretera del Canyet, s/n, 08916 Badalona, Spain radiological, or histological parameters in our patient. Despite treatment, patient persists with incapacitating bone pain requiring opioids and an electrical wheelchair. Orthopedic surgery has been discouraged.

\section{Discussion}

FIO is an extremely rare acquired progressive incapacitating bone disease of poorly understood etiology [1]. The underlying pathology appears to be a defective bone mineralization and abnormal bone collagen morphology that radically replaces normal lamellar bone [2]. The clinical manifestations include bone pain, multiple fractures, and bone deformities, leading to immobility and bedridden. Paraproteinemia is observed in a one-third of cases [2]. Radiographic findings commonly seen in FIO include axial and appendicular sclerotic changes, pseudofractures, vertebral fractures, axial osteopenia, cortical thinning of long bones, and formation of ectopic bone in the soft tissues. In advanced disease, the "fishnet" trabecular pattern is frequently observed showing marked reduction of bone density, loss of trabeculae and coarsening of the remaining trabeculae $[1,2]$. Whereas histomorphometric bone biopsy analysis shows severe abnormal bone mineralization and increased bone turnover resembling osteomalacia, the final diagnosis is performed by polarized light microscopy with evidence of non-lamellar bone or collagen birefringence (although the latter can be observed in collagen diseases like osteogenesis imperfecta type VI) [1, 2]. Prognosis is poor due to delayed diagnosis and the progressive nature of the disease $[1,2]$. Treatment with glucocorticoids, bisphosphonates, melphalan, plasmapheresis, and recombinant growth hormone therapy has been tried previously with variable success [1-3]. Further research is needed to identify pathogenic factors and develop targeted therapeutic options. 


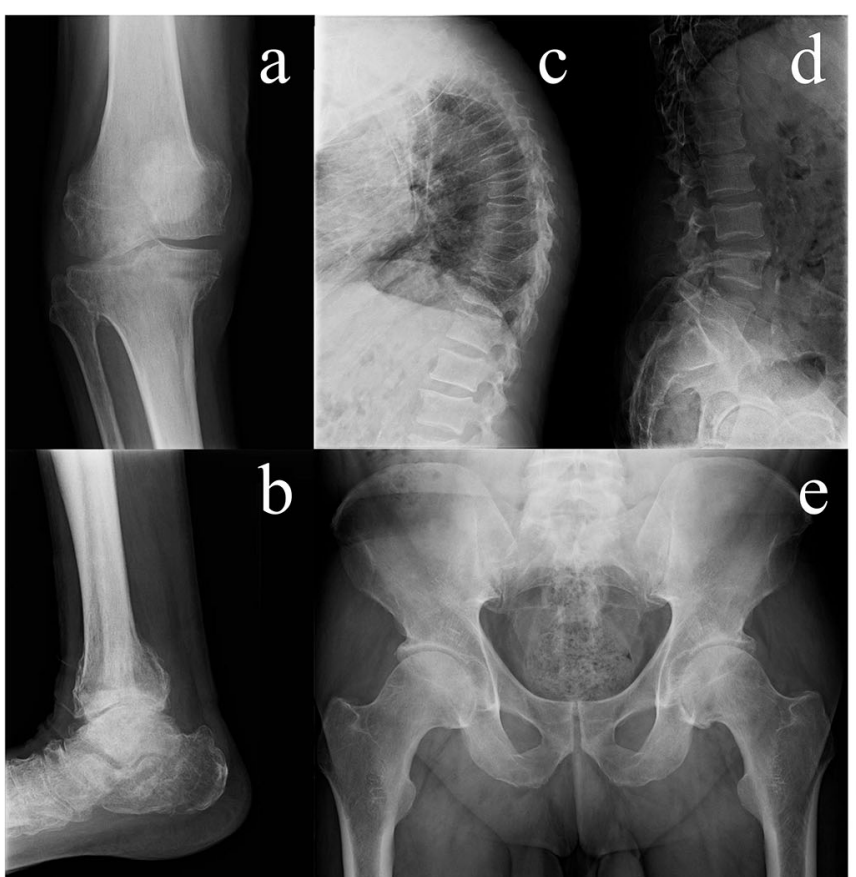

Fig. 1 a Radiograph of the right knee with varus deformity, signs of osteoarthritis, thickened trabecular bars seen along the inferior femoral and superior tibial metaphyses, and pseudo-fractures. b Radiograph of ankle with pseudo-fractures, fracture-related deformities, cortical thinning, osteoarthritis, and noticeable osteoporosis of the calcaneus. c, d Lateral thoracic and lumbar radiographs showing thoracic kyphosis on top of progressive flattening of the thoracic vertebral bodies, upper and

Supplementary Information The online version contains supplementary material available at https://doi.org/10.1007/s10067-021-05708-5.

Author contributions AP-E and LG draft the publication and all authors were involved in revising the article critically. The final version of the manuscript was seen and approved by all authors.

\section{Declarations}

Ethics approval and consent to participate All procedures performed in this study were in accordance with the ethical standard of Hospital Universitari Germans Trias i Pujol and with the 1964 Helsinki Declaration and its later amendments. IRB approval was not required.

Consent for publication The patient has given his consent for the publication of this manuscript.

Disclosure None

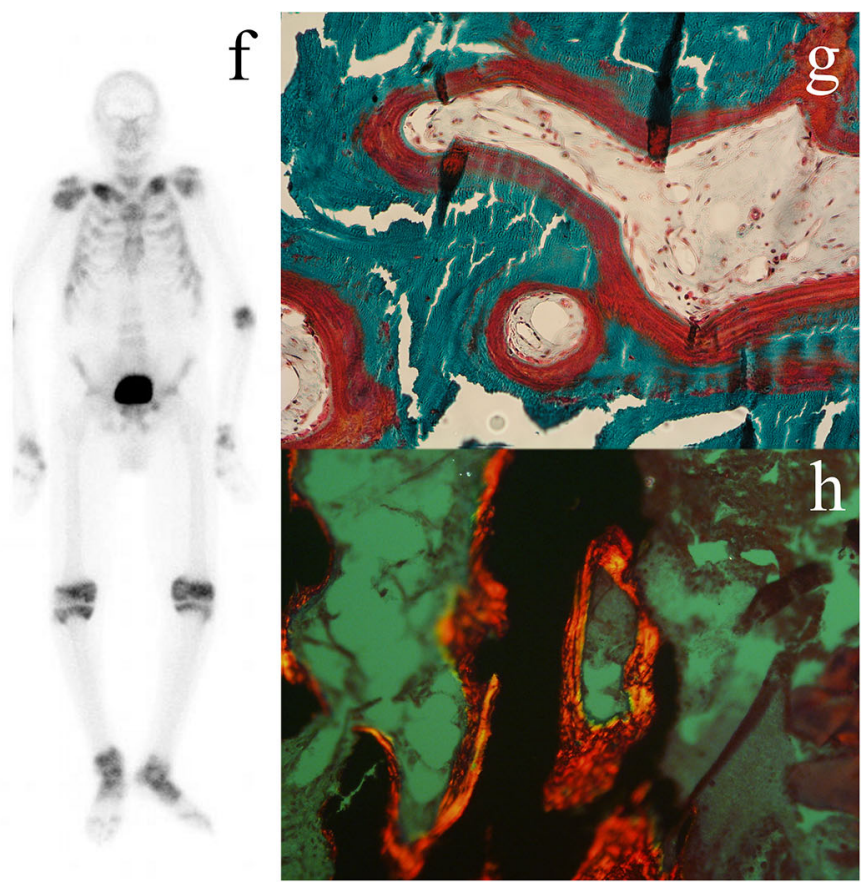

lower vertebral platform sclerosis, and trabecular osteopenia. e Anteroposterior pelvis radiograph showed narrowed joint space with signs of mild impingement of the hips. f Bone scintigraphy showed multiple uptake locations with the exception of the skull. $\mathrm{g}$ A Masson trichrome stain of undecalcified bone biopsy showed extensive nonmineralized osteoid accumulation. h Polarized light microscopy revealed disorganized non-mineralized osteoid with irregular distribution

\section{References}

1. Dhaliwal R, Dhiman V, Rao SD, Bhadada SK (2019) Fibrogenesis imperfecta ossium: clinical approach to diagnosis and management of a rare skeletal disorder. J Clin Endocrinol Metab 14:jc.201801180. https://doi.org/10.1210/jc.2018-01180

2. Bhadada SK, Dhaliwal R, Dhiman V, Rao SD (2019) Fibrogenesis imperfecta ossium. Calcif Tissue Int 104:561-569. https://doi.org/ 10.1007/s00223-019-00547-8

3. Bakos B, Lukáts Á, Lakatos P, Győri G, Tremmel A, Takács I (2014) Report on a case of fibrogenesis imperfecta ossium and a possible new treatment option. Osteoporos Int 25(5):1643-1646. https://doi. org/10.1007/s00198-014-2675-8

Publisher's note Springer Nature remains neutral with regard to jurisdictional claims in published maps and institutional affiliations. 